Originally published as:

R. Schlack, H. Hölling, M. Erhart, F. Petermann, U. Ravens-Sieberer. Elterliche Psychopathologie, Aggression und Depression bei Kindern und Jugendlichen (2010) Kindheit und Entwicklung, 19 (4), pp. 228-238.

DOI: $10.1026 / 0942-5403 / a 000030$

This is an author manuscript.

The definitive version is available at: http://psycontent.metapress.com/ 


\title{
Elterliche Psychopathologie, Aggression und Depression bei Kindern und Jugendlichen
}

\author{
Robert Schlack ${ }^{1}$, Heike Hölling ${ }^{1}$, Michael Erhart ${ }^{2}$, Franz Petermann ${ }^{3}$, Ulrike Ravens-Sieberer ${ }^{2}$ \\ ${ }^{1}$ Robert Koch-Institut Berlin \\ ${ }^{2}$ Forschungssektion „Child Public Health“ des Universitätsklinikums Hamburg-Eppendorf \\ ${ }^{3}$ Zentrum für Klinische Psychologie und Rehabilitation der Universität Bremen
}

\section{Zusammenfassung}

Aggressiv-dissoziales Verhalten und depressive Symptome treten bei Kindern und Jugendlichen auch gemeinsam auf. Eine solche Komorbidität zweier unterschiedlicher Störungsbilder kann oft durch einen gemeinsamen Risikofaktor erklärt werden. Aus der bevölkerungsbezogenen BELLA-Studie wurde aus insgesamt 1356 Teilnehmern im Altern von elf bis 17 Jahren (51.6 \% Jungen) eine Risikogruppe aggressiver Kinder und Jugendlicher ( $n=422 ; 68.7 \%$ Jungen) identifiziert und untersucht, ob und inwieweit die Beziehungen zwischen elterlicher Psychopathologie und einer der beiden Störungen von der jeweils anderen Störung vermittelt werden. Hierzu wurden alters- und geschlechtsadjustierte Mediatoranalysen durchgeführt. Die Analysen wurden stratifiziert, je nachdem ob sich die Kinder und Jugendlichen als vorwiegend proaktiv ( $n=145 ; 52.4 \%$ Jungen), reaktiv $(n=$ $173 ; 76.3 \%$ Jungen) oder reaktiv-proaktiv ( $n=104 ; 78.8 \%$ Jungen) aggressiv einstuften. Für alle Risikogruppen mediierte sowohl aggressiv-dissoziales Verhalten den Pfad auf Depression als auch Depression den Pfad auf aggressiv-dissziales Verhalten. Die Varianzaufklärung der Modelle war für proaktiv- und reaktiv-aggressive Jugendliche deutlich höher, wenn Depression den Pfad von elterlicher Psychopathologie auf aggressiv-dissoziales Verhalten mediierte. Für reaktiv-proaktiv aggressive Kinder und Jugendliche war dieses Verhältnis umgekehrt. Die Ergebnisse werden vor dem Hintergrund aktueller Ergebnisse und Komorbiditätskonzepte diskutiert.

Schlüsselwörter: Aggression, Depression, elterliche Psychopathologie, proaktiv-aggressives Verhalten, reaktiv-aggressives Verhalten

In epidemiologischen Studien treten häufig aggressiv-dissoziale Verhaltensweisen und depressive Symptome bei Kindern und Jugendlichen gemeinsam auf (Angold \& Costello, 1993; Beyers \& Loeber 2003; Kopp \& Beauchaine, 2007). Heranwachsende mit kombinierten Verhaltensauffälligkeiten und Depression haben ein höheres Risiko für ungünstige klinische Verläufe ihrer Störung (Beyers \& Loeber, 2003; Fombonne et al., 2001; Groen \& Petermann, 2008; Marmorstein \& lacono, 2003), für erneute Krankheitsphasen nach erfolgter Behandlung (Rohde et al., 2001), Suizidversuche (Fombonne et al., 2001) sowie kriminelles Verhalten im Erwachsenenalter (Sourander et al., 2007). Aber auch beim Bildungserfolg sowie bei sozialer und allgemeiner Funktionsfähigkeit scheinen Jugendliche mit komorbider depressiver und aggressiver Störung schlechter abzuschneiden als Jugendliche mit jeweils nur einer der beiden Störungen (Ezpeleta et al., 2006; Ingoldsby et al., 2006; Marmorstein \& lacono, 2003).

Über die Natur der Komorbidität dieser empirisch und konzeptionell unterschiedlichen Störungsbilder (externalisierend und internalisierend) gibt es keinen gesicherten Konsens (vgl. Angold et al., 1999; Scheithauer \& Petermann, 2010). Studien, die die statistische Komorbidität untersuchten, kamen zu dem Ergebnis, dass Aggression deutlich häufiger bei Kindern mit depressiver Störung auftritt als umgekehrt bei Kindern mit Aggression eine Depression diagnostiziert werden muss (Angold \& Costello, 1993; McConaughy \& Achenbach, 1994). Allerdings zeigten Ergebnisse von Längsschnittstudien, dass aggressiv-dissoziales Verhalten im zeitlichen Verlauf häufig vor einer Depression auftritt (Capaldi, 1992; Panak \& Garber, 1992) und gleichsam die Rolle eines Risikofaktors für diese einnimmt. Als Mediator zwischen aggressivem Verhalten und depressiven Stimmungen wurde hierbei vor allem geringere soziale Kompetenz aggressiver Kinder (Cole et al., 1997) sowie die Zurückweisung durch Gleichaltrige (Morrow et al., 2006) empirisch untersucht und diskutiert. 
In Folge ihres externalisierenden Verhaltens erleben aggressive Kinder mehr Ablehnung durch Gleichaltrige bzw. vermehrt Misserfolge in anderen sozialen Konstellationen wie Familie oder Schule (Petermann et al., 2008; Scheithauer et al., 2003). In Anlehnung an Lewinsohns integrative Theorie der unipolaren Depression können ein Mangel an sozialen Fertigkeiten und geringe soziale Verstärker depressive Stimmungen hervorrufen und eine depressive Symptomatik auslösen; doch ist die Studienlage hier keineswegs eindeutig (vgl. Petermann, 2002). So konnten die Ergebnisse von Panak und Garber (1992), dass Zurückweisung durch Gleichaltrige den Pfad von aggressivem Verhalten zu depressiven Symptomen mediierte, nicht bestätigt werden (Kiesner, 2002). Beyers und Loeber (2003) untersuchten in einer Längsschnittstudie Verlaufs- und Entwicklungsaspekte für die Zusammenhänge von Delinquenz und depressiven Stimmungen bei männlichen Jugendlichen im Alter von 13.5 bis 17.5 Jahren. Die Autoren fanden bei statistischer Kontrolle initialer Risikofaktoren und unter Berücksichtung zeitlich variierender Begleitfaktoren wie Alterseffekten oder aktueller Depression/Delinquenz, dass sowohl Depression gleichzeitig auftretende Delinquenz sowie deren Verlauf und deren Schwere voraussagte, wie umgekehrt Delinquenz depressive Stimmungen. Allerdings hatten ihren Ergebnissen zufolge depressive Stimmungen eine stärkere Bedeutung für den Verlauf unterschiedlicher Formen von Delinquenz als umgekehrt Delinquenz auf den Verlauf depressiver Stimmungen. Die Tatsache allein, dass eine Störung der anderen zeitlich vorausgeht, lässt jedoch noch nicht auf eine Kausalität schließen. Möglich ist ebenso eine wahre Komorbidität, d.h. eine Komorbidität Aggression/ Depression als eigenständiges Störungsbild (vgl. Kopp \& Beauchaine, 2007). Eine mögliche Erklärung wahrer Komorbidität ist nach Caron und Rutter (1991) die Existenz gemeinsamer Risikofaktoren zweier Störungen.

Ein potenzieller Kandidat für einen solchen Risikofaktor mit Blick auf die Komorbidität von aggressivem Verhalten und depressivem Symptomen ist die elterliche Psychopathologie (vgl. Jeske et al., 2009;Wiegand-Grefe et al., 2009). Eine Studie von Cohen und Kollegen (1990) zeigte, dass, neben Scheidung und Wiederheirat der Eltern, elterliche Psychopathologie sowohl mit internalisierenden als auch externalisierenden Problemen ihrer Kinder assoziiert war. Eltern verhaltensauffälliger Kinder zeigen häufig selbst aggressiv-dissoziales Verhalten oder Züge einer antisozialen Persönlichkeitsstörung (Frick et al., 1992; Marmorstein \& lacono, 2004). Eine neuere Studie zeigte, dass speziell antisoziale Persönlichkeitsstörungen bei Vätern und depressive Episoden beiMüttern mit erhöhtem Risiko sowohl für aggressiv-dissoziales Verhalten, Depressionen als auch für das komorbide Auftreten beider Störungen verbunden waren (Kopp \& Beauchaine, 2007). Wenn Eltern sowohl Verhaltensstörungen als auch eine Major Depression aufwiesen, waren kombinierte Verhaltensauffälligkeiten und Depressionen bei ihren Kindern häufiger als wenn die Eltern ausschließlich eine Major Depression aufwiesen (Hirshfeld- Becker et al., 2008).

Durch die elterliche Psychopathologie kommt es zu Fehlanpassungen bei Kindern und Jugendlichen und einer beeinträchtigten Erziehungsfähigkeit der Eltern (z. B. Vernachlässigung, inkonsistenter Erziehungsstil, dysfunktionale Disziplinierungspraktiken oder geringeres Kompetenzerleben; siehe Berg-Nielsen et al., 2002; Kötter et al., 2010; Petermann et al., 2002). Ein Reihe von Studien zeigte, dass depressive Mütter gegenüber ihren Kindern häufiger kritisch eingestellt sind, negative Stimmungen und zurückweisendes Verhalten zeigen (Goodman et al., 1994; Murray et al. 1993), stärker selbstzentriert sind sowie geringere Sensibilität für die Bedürfnisse ihrer Kinder an den Tag legen und zu inkonsistentem Erziehungsverhalten neigten (Conger et al., 1992; Koglin \& Petermann, 2008). Antisoziale Persönlichkeitszüge (von Müttern) waren dagegen mit mangelnder Responsivität gegenüber den Kindern verbunden (Cassidy et al., 1996). Allerdings sind auch genetische Ursachen bzw. Gen- Umweltinteraktionen denkbar. In einer Zwillings-/Geschwisterstudie ließen sich $45 \%$ der Kovariation zwischen Depression und aggressiv-dissozialem Verhalten auf genetische Ursachen zurückführen ((O’Connor et al., 1998).

In der Aggressionsforschung wird zwischen proaktiver und reaktiver Aggression unterschieden (Card \& Little, 2006; Kruse \& Petermann, 2010; Polman et al., 2007). Unter proaktiver Aggression wird ein geplantes, zielorientiertes (instrumentelles) Verhalten verstanden, während reaktiv-aggressives Verhalten eher eine spontan-situative Reaktion auf eine tatsächliche oder vermeintliche Bedrohung darstellt, die häufig mit dem Gefühl von Ärger verbunden ist (vgl. auch Petermann\& Petermann, 2010). Das Konzept der proaktiven Aggression (auch als „kaltblüti- ge“ Aggression bezeichnet) basiert auf der sozial-kognitiven Lerntheorie (Bandura, 1973, 1986). Dort wird Aggression als Ergebnis hoher Selbstwirksamkeitserwartungen bezüglich aggressiven Verhaltens sowie vorteilhafter Ergebniserwartungen verstanden, die durch operantes Konditionieren und Modelllernen erlernt werden. Die Konzeption kann als empirisch gesichert gelten (vgl. z. B. Crick \& Dodge, 1996; Egan et al., 1998; Helmsen \& Petermann, 2010). Reaktive Aggression bezieht sich auf das Frustrations- 
Aggressions-Modell (Berkowitz, 1993). In diesem Modell ist aggressives Verhalten eine feindseligärgerliche Reaktion auf eine Frustrationen oder eine tatsächliche oder vermeintliche Provokation. Studien, die die Beziehungen von proaktiver und reaktiver Aggression mit Problemen psychosozialer Anpassung untersuchten, ergaben, dass proaktive Aggression vor allem aggressives und dissoziales Verhalten voraussagte, während reaktive Aggression vornehmlich mit depressiven Symptomen assoziiert war (Card\& Little, 2006; Fite et al., 2009; 2010; Vitaro et al., 2002).

\section{Ziel der Studie}

Es sollen zwei unterschiedliche Pfade elterlicher Psychopathologie auf Depression und aggressivdissoziales Verhalten vergleichend untersucht werden. Wie oben dargestellt, kann elterliche Psychopathologie als gemeinsamer Risikofaktor für depressive Symptome ebenso wie für aggressivdissoziales Verhalten angesehen werden. Gleichzeitig liegen widersprüchliche empirische Ergebnisse zu ätiologischen Beziehungen zwischen Aggression und Depression vor. Die Studienfrage lautet: „Wird die Beziehung von elterlicher Psychopathologie und aggressiv- dissozialem Verhalten durch depressive Symptome mediiert?" und alternativ: „Wird die Beziehung von elterlicher Psychopathologie und depressiven Symptomen von aggressiv-dissozialem Verhalten mediiert?" Die Studie wurde mit Daten von Kinder und Jugendliche im Alter von elf bis 17 Jahren aus der bevölkerungsbezogenen BELLA-Studie durchgeführt, die Angaben zum Ausmaß ihrer proaktiven und reaktiven Aggressionsbereitschaft gemacht hatten. Alle Analysen wurden danach stratifiziert, ob die Kinder und Jugendlichen anhand ihrer Selbstbeurteilung als vorwiegend proaktiv-, vorwiegend reaktiv-, vorwiegend reaktiv-proaktiv aggressiv oder nichtaggressiv eingestuft werden konnten.

Auf der Grundlage der oben referierten Ergebnisse wurden folgende Hypothesen formuliert (vgl. auch Abb. 1):

Hypothese 1: Es wurde erwartet, dass proaktiv-aggressive Jugendliche höhere Werte hinsichtlich aggressiv-dissozialemVerhaltens aufweisen als reaktiv-aggressive. Für diese wurden dagegen erwartet, dass sie höhere Werte auf der Depressionsskala aufweisen (Hypothese 2). Für die reaktivproaktive Gruppe wurde vermutet, dass diese besonders hohe Werte sowohl auf derDepressionsskala als auch auf der Skala für externalisierendes Verhalten aufweist (Hypothese 3). Die Hypothesen zu den Mediatiormodellen lauteten: Hypothese 4: Der negative Effekt von elterlicher Psychopathologie auf aggressiv-dissoziales Verhalten wird durch eine Zunahme der depressiven Symptomatik mediiert. Eine Bestätigung dieser Hypothese wäre eine Unterstützung für die Annahme, dass Depression eine ätiologische Bedeutung für aggressiv-dissoziales Verhalten besitzt. Hypothese 5: Der negative Effekt von elterlicher Psychopathologie auf die depressive Symptomatik wird durch die Zunahme aggressiven Verhaltens mediiert. Eine Bestätigung dieser Hypothese könnte hingegen als Bekräftigung der Annahme gelten, dass aggressiv-dissoziales Verhalten in einer ätiologischen Beziehung zu Depression steht.

\section{Methode}

\section{Stichprobe}

Die Daten der vorliegenden Untersuchung stammen aus der BELLA-Studie (Ravens-Sieberer et al., 2007). Die BELLA-Studie ist ein Zusatzmodul zur psychischen Gesundheit der bevölkerungsbezogenen KiGGS-Studie (Kurth et al., 2008), die von 2003 bis 2006 mit 17.641 Kindern und Jugendlichen und ihren Eltern in insgesamt 167 repräsentativ ausgewählten Städten und Gemeinden der Bundesrepublik Deutschland mit einer Responserate von $66.6 \%$ durchgeführt wurde. Die Teilnehmer der BELLA-Studie wurden aus den Teilnehmern der bundesweit repräsentativen KiGGS-Studie randomisiert rekrutiert. Von 4199 angesprochenen Familien nahmen $68 \%$ (1389 Mädchen und 1474 Jungen und ihre Eltern) an der Modulstudie teil. Für jede Familie wurde ein Elternteil (Vater oder Mutter) sowie ab elf Jahren auch die Kinder und Jugendlichen selbst mit einem computergestützten, standardisierten Telefoninterview (CATI) sowie mit einem postalischen schriftlichen Fragebogen befragt. Für Details der Studie kann auf Ravens-Sieberer und Kurth (2008) verwiesen werden. Die aktuelle Stichprobe umfasst 1356 Jungen und Mädchen im Alter von elf bis 17 Jahren (Durchschnittsalter: $M=14.39 ; S D=1.98$ ) mit vollständigen Angaben auf allen herangezogenen Instrumenten (listenweiser Missing-Ausschluss). 


\section{Erhebungsinstrumente}

Depressive Symptome. Zur Erfassung depressiver Symptome wurde die deutsche Übersetzung der Centre of Epidmiological Studies Depression Scale for Children (CES-DC) in Form eines Eltern- und Selbstberichts eingesetzt (Barkmann et al., 2008). Die CES-DC wurde als Screening von Symptomen verschiedener depressiver Störungsbilder in bevölkerungsbezogenen Studien entwickelt. Ihre 20 Items lassen sich zu einem Gesamtwert globaler Depressivität aufsummieren. Für diese Analyse wurde der Elternbericht herangezogen. Die interne Konsistenz für die vorliegende Stichprobe betrug a $=.83$.

Externalisierendes Verhalten. Erhoben mit den Skalen „Aggressives Verhalten“ und „Dissoziales Verhalten“ der Child Behavior Checklist (CBCL von Achenbach (1991); Elternfragebogen). Die insgesamt 33 Items der beiden Skalen lassen sich zu einem Wert „Externalisierendes Verhalten“ zusammenfassen, der für diese Analysen verwendet wurde. Cronbachs a lag für die vorliegende Stichprobe bei .91 .

Reaktive und proaktive Aggression. Diese Aggressionsformen wurden mit zwei Skalen mit jeweils drei Items zu instrumentellem und expressiv-situativem Gewalthandeln erfasst (Heitmeyer, 2002, persönlicheMitteilung; Schlack \& Hölling, 2007). Mit Blick auf proaktive Aggression lauteten die Items z.B. „Um mich durchzusetzen, muss ich manchmal auch gewalttätig werden.", für reaktive Aggression z.B. „Wenn mich jemand persönlich beleidigt, kann es inm passieren, dass er sich eine einfängt." Die Items waren jeweils auf einer vierstufigen Antwortskala von „Trifft überhaupt nicht“ bis „Trifft genau zu“ zu beantworten. Die internen Konsistenzen lagen für die Skala „Proaktive Aggression“ bei a .60, für Skala „Reaktive Aggression“ bei $\alpha=.71$. Die Interkorrelation der Skalen für proaktive und reaktive Aggression lag bei $r=.31$, was als Hinweis auf eine hinreichende diskriminante Validität betrachtet werden kann. Anhand ihrer Selbstbeurteilungen der reaktiven und proaktiven Aggression wurden insgesamt 422 Jungen und Mädchen als Risikogruppe aggressiver Kinder identifiziert. Das Urteil "vorwiegend proaktiv-aggressiv" ( $n=145 ; 52$ \%Jungen) wurde vergeben, wenn der Summenwert auf der Skala „Proaktive Aggression“" mehr als eine Standardabweichung über dem Mittelwert lag und gleich dem oder unterhalb des Mittelwerts plus einer Standardabweichung auf der Skala „Reaktive Aggressivität“. Umgekehrt wurde für „Vorwiegend reaktiv-aggressiv“ ( $n=173 ; 76$ \% Jungen) verfahren. Kinder und Jugendliche, deren Summenwerte jeweils mehr als eine Standardabweichung über demMittelwert sowohl der Skala „Proaktive Aggression“ als auch der Skala „Reaktive Aggression“ lag, wurden als reaktiv-proaktiv aggressiv eingestuft ( $n=104 ; 78.8 \%$ Jungen).

Elterliche Psychopathologie. Die globale elterliche Psychopathologie wurde mit einer Kurzform der SCL-90-R (Franke, 2002) im schriftlichen Elternfragebogen erhoben. Die Kurzform SCL-K-9 erfasst mit jeweils einem Item Somatisierung, Zwanghaftigkeit, Unsicherheit im Sozialkontakt, Ä ngstlichkeit, Aggressivität, phobische Angst, paranoides Denken und Psychotizismus. In einer Analyse mit 8581 Patienten mit psychischen Erkrankungen wurde die psychometrische Qualität der SCL-90-R und ihrer Kurzformen sowie ihre Geeignetheit als Screeninginstrument für einen breiten Bereich psychopathologischer Symptomatik überprüft und bestätigt (Prinz et al., 2008). Die interne Konsistenz der SCL-K-9 in dieser Studie betrug $\alpha=.83$.

\section{Statistische Analysen}

Unterschiede zwischen den verschiedenen Aggressionstypen: proaktiv-, reaktiv-, reaktiv-proaktiv- und nichtaggressiv bezüglich der Variablen externalisierendes (= aggressivdissoziales) Verhalten, Depression und elterliche Psychopathologie wurden mit einer einfaktoriellen multivariaten Varianzanalyse (MANOVA) und anschließenden univariaten Varianzanalysen (ANOVA) nach demAllgemeinen Linearen Modell überprüft. Für die Bestimmung von paarweisen Gruppenunterschieden wurden Post-Hoc-Tests gerechnet (T2- und Scheffé-Tests).

Zur Schätzung der vermuteten Mediationseffekte wurden Mediatoranalysenmit der BootstrapResampling- Methode durchgeführt. Bootstrap-Resampling ist eine Simulationsmethode, bei der wiederholt Stichproben (mit Zurücklegen) aus den Originaldaten gezogen werden (mindestens 1000). In jeder einzelnen dieser Stichproben wird der indirekte Effekt $(a \times b)$ bestimmt. Auf diese Weise wird eine empirische Stichprobenverteilung des indirekten Effekts $(a \times b)$ erzeugt, aus der unmittelbar die Konfindenzintervalle bestimmt werden können. Diese erhält man, indem man die so erzeugten empirischen indirekten Effektschätzer der Größe nach sortiert. Die obere Grenze eines 95 \%Konfidenzintervalls des indirekten Effekts wird bestimmt, indem man die oberen $2.5 \%$, die untere, 
indem man die unteren $2.5 \%$ von dieser sortierten Verteilung abschneidet. Bei 1000 BootstrapResamples wäre die obere Konfidenzgrenze damit der 976.Wert, die untere Konfidenzgrenze der 25. Wert dieser Stichprobenverteilung. Ein signifikanter (ggf. partieller) Mediationseffekt auf dem 0.05Niveau liegt dann vor, wenn das Konfidenzintervall für den indirekten Effekt denWert Null nicht umschließt (vgl. Preacher \& Hayes, 2004, 2008; Hayes, 2009).Wenn der direkte Effekt (c'-Pfad) seine Signifikanz verliert, kann von vollständiger Mediation gesprochen werden. BootstrapKonfidenzintervalle sind nicht symetrisch (siehe Hayes, 2009). ImUnterschied zur klassischen Mediatoranalyse nach Baron und Kenny (1986) besitzt diese Methode eine höhere statistische Power und setzt keine Normalverteilung des indirekten Effekts für dessen Signifikanzbestimmung voraus (Hayes, 2009).

Preacher und Hayes (2008) stellen ein SPSS-Makro für die Überprüfung multipler Mediatormodelle zur Verfügung, dass hier verwendet wurde, weil es die Möglichkeit bietet, für Kontrollvariablen zu adjustieren. Das Makro erlaubt keine Verwendung gewichteter Daten oder die Berücksichtigung komplexer Stichprobenstrukturen, wie sie den BELLA-Daten zu eigen ist. Daher wurden konsequenterweise auch die sonstigen Analysen ohne Berücksichtigung des BELLA-Designgewichts durchgeführt. Dadurch ist zum einen die Repräsentativität eingeschränkt, zum anderen hat dies zur Folge, dass Signifikanzen leicht überschätzt werden. Dies wurde jedoch zugunsten der Verwendung einer höherwertigen statistischen Methode in Kauf genommen. Um das Ausmaß möglicher Verzerrungen abzuschätzen, wurden vergleichende Regressionsanalysen jeweils mit und ohne Gewichtung durchgeführt. Hierbei zeigte sich jedoch, dass die Abweichungen gering und für die Signifikanzprüfungen praktisch unbedeutend waren, jedoch mit sinkender Stichprobengröße zunahmen. Insofern sind insbesondere die Analysen für die reaktiv-proaktiv aggressive Gruppe vorsichtig zu interpretieren. Alle Analysen wurden mit SPSS (PASW) 17.0 durchgeführt.

\section{Ergebnisse}

Tabelle 1 zeigt die deskriptiven Statistiken und die Korrelationen der Variablen für die Mediatormodelle. Alle Modellvariablen korrelierten signifikant in moderater Höhe, in allen Gruppen in der erwarteten Richtung. Erhöhte Werte bei aggressiv-dissozialem Verhalten gingen einher mit erhöhten Werten hinsichtlich depressiver Stimmung. Sowohl aggressiv-dissoziales Verhalten als auch depressive Symptome korrelierten in allen Gruppen mit elterlicher Psychopathologie wobei für proaktiv- und reaktiv-aggressive Personen die Korrelation von elterlicher Psychopathologie mit externalisierendem Verhalten höher war, während für reaktiv-aggressive Jugendliche die Korrelation mit Depression höher ausgeprägt war.

Die einfaktorielle multivariaten Varianzanalyse (MANOVA) ergab signifikante Gruppenunterschiede zwischen proaktiv-, reaktiv-, reaktiv-proaktiv- und nichtaggressiven Kindern und Jugendlichen in den Variablen aggressiv-dissozialesVerhalten, Depression und elterliche Psychopathologie (Wilks-Lambda $\left.=.901 ; F_{(3,1350)}=15.62 ; p<.001\right)$. Die nachfolgenden univariaten Varianzanalysen (ANOVA) waren für alle drei Variablen ebenfalls signifikant (Externalisierendes Verhalten: $F_{(3,1352)}=42.38 ; p<.001$; Depression: $\mathrm{F}_{(3,1352)}=8.17 ; p<.001$; elterliche Psychopathologie: $\left.\mathrm{F}_{(3,1352)}=8.46 ; p<.001\right)$. Post-HocVergleiche ergaben, dass proaktivaggressive Kinder entgegen der Erwartung signifikant höhere Depressionswerte aufwiesen als sämtliche anderen Gruppen, die sich untereinander hierin nicht unterschieden. Damit sind die Hypothesen 1 und 2 nicht bestätigt. Die reaktiv-proaktiv aggressive Gruppe wies hinsichtlich externalisierender (= aggressiv-dissozialer) Störungen die bei weitem höchsten Werte auf und unterschied sich signifikant von der proaktiv- und reaktiv-aggressiven Risikogruppe, welche sich untereinander nicht unterschieden. Damit ist Hypothese 3 teilweise bestätigt. Nichtaggressive Kinder hatten hier erwartungsgemäß signifikant niedrigere Werte als Angehörige aller Risikogruppen. Bei der elterlichen Psychopathologie unterschieden sich reaktiv- und reaktiv-proaktiv aggressive Jugendliche signifikant von nichtaggressiven, untereinander unterschieden sich die Risikogruppen diesbezüglich nicht.

\section{Ergebnisse der Mediatoranalysen}

Abbildung 1 skizziert die in den Mediatoranalysen getesteten Zusammenhänge. Alter und Geschlecht wurden jeweils als Kontrollvariablen in die Modelle aufgenommen. Die Modellinformationen für alle Mediatoranalysen finden sich in Tabelle 2, die Ergebnisse der Mediatoranalysen in Tabelle 3. In den die Hypothese 4 betreffenden Analysen war aggressiv-dissoziales Verhalten die abhängige Variable 
und Depression der Mediator (Tab. 3). Für die Gruppen nichtaggressiv (95\% KI: 0.0173-0.0390), proaktiv-aggressiv (95\% KI: $0.0181-0.900)$, reaktivaggressiv (95\% KI: $0.0248-0.1083$ ) und reaktivproaktiv aggressiv (95\% KI: 0.0094- 0.1237) war Depression ein signifikanter Mediator für die Beziehung von elterlicher Psychopathologie und aggressiv-dissozialem Verhalten. In den die Hypothese 5 betreffenden Analysen (Depression als abhängige Variable und aggressiv-dissoziales Verhalten als unabhängige Variable) waren die Mediationseffekte für aggressiv-dissoziales Verhalten ebenfalls sämtlich signifikant: nichtaggressive Gruppe: $95 \% \mathrm{KI}: 0.230-0.0489$; proaktiv-aggressive Gruppe: $95 \%$ KI: $0.0288-0.1254$; reaktiv-aggressive Gruppe: $95 \% \mathrm{KI}$ : 0.0332- 0.1279; reaktivproaktiv aggressive Gruppe: $95 \%$ KI: 0.0037-0.0487. Für die reaktiv-aggressive Gruppe war der direkte Effekt (c'-Pfad) insignifikant, so dass hier von vollständiger Mediation gesprochen werden kann. Die Varianzaufklärung für die Hypothese- 5-Modelle mit aggressiv-dissozialem Verhalten als abhängiger Variable war jedoch deutlich geringer hinsichtlich der nichtaggressiven, proaktiv- sowie reaktivaggressiven Jugendlichen. Für die reaktiv-proaktiv aggressiveGruppe lag diese jedoch geringfügig höher als bei den Hypothese-4-Modellen. Damit ist sowohl Hypothese 4 als auch Hypothese 5 für alle Gruppen bestätigt. Da diese Ergebnisse weiter differenziert werden sollten, wurden zusätzliche Analysen durchgeführt, um die Unterschiede in der Varianzaufklärung zu erhellen. Für jede Gruppe wurden vier einfache alters- und geschlechtsadjustierte Regressionsanalysen gerechnet, in denen aggressiv-dissoziales Verhalten auf elterliche Psychopathologie und Depression sowie Depression auf elterliche Psychopathologie und aggressiv-dissoziales Verhalten rückbezogen wurde. Das Signifikanzniveau wurde auf $p<.003(.05 / 16)$ festgelegt (Bonferroni-Korrektur für multiples Testen).

Die Ergebnisse zeigten, dass in der proaktiv-aggressiven Gruppe elterliche Psychopathologie $19.3 \%$ der Varianz von aggressiv-dissozialem Verhalten aufklärte, aber nur $9.0 \%$ von Depression. Wurde Depression zur Vorhersage von aggressiv-dissozialem Verhalten herangezogen, war die Varianzaufklärung mit 15.9 \% annä- hernd genauso hoch wie im umgekehrten Fall (16.1\%). Ein ähnliches Bild zeigte sich für die reaktiv-aggressive Gruppe: elterliche Psychopathologie klärte $20.9 \%$ der Varianz von externalisierendem Verhalten auf aber nur $8.1 \%$ von Depression, die Vorhersage von Depression klärte $20.8 \%$ der Varianz von aggressiv-dissozialem Verhalten, umgekehrt aggressivdissoziales Verhalten 18.9 \% der Varianz von Depression auf. Ein anderes Bild zeigte sich für die reaktiv-proaktiv aggressive Gruppe. Hier war die Varianzaufklärung nicht nur insgesamt geringer, sondern auch die Richtung vertauscht: elterliche Psychopathologie klärte nur $13.3 \%$ der Varianz von externalisierendem Verhalten, jedoch $15.9 \%$ von Depression auf. Die Vorhersage aggressivdissozialen Verhaltens durch Depression klärte $10.4 \%$ der Varianz auf, im umgekehrten Fall betrug sie $8.7 \%$. Die Gruppe der nichtaggressiven Kinder und Jugendlichen verhielt sich auf niedrigerem Niveau analog der proaktiv- und reaktiv-aggressiven Gruppe und wird hier nicht weiter beschrieben. Alle Ergebnisse waren auf dem festgelegten Signifikanzniveau statistisch bedeutsam.

\section{Diskussion}

In dieser Studie wurden die Beziehungen von elterlicher Psychopathologie, aggressiv-dissozialem Verhalten und Depressivität bei Kindern und Jugendlichen untersucht, die aufgrund ihrer Selbstbeurteilungen als vorwiegend proaktiv-, reaktiv- oder reaktiv-proaktiv aggressiv klassifiziert wurden.

Die deskriptiven Analysen zeigen, dass sich Kinder und Jugendliche mit proaktiv-, reaktiv-, reaktivproaktiv aggressiven Einstellungen und nichtaggressive in ihrem Risikoprofil hinsichtlich elterlicher Psychopathologie nicht unterschieden und hinsichtlich depressiver Stimmungen nur bei proaktivaggressiven Kindern signifikant erhöhte Werte gefunden wurden. Dieser Befund überrascht, denn hier wäre angesichts der eingangs dargestellten Befunde zu erwarten gewesen, dass reaktiv-aggressive Kinder höhere Depressionswerte aufweisen. Dass Kinder und Jugendliche mit aggressiven Einstellungen generell höhere Werte hinsichtlich aggressiv-dissozialen Verhaltens aufweisen, entspricht der Erwartung. Auffällig sind jedoch die extrem erhöhten Werte für aggressiv- dissoziales Verhalten bei der reaktiv-proaktiv aggressiven Gruppe.

Die Ergebnisse der Mediatoranalysen zeigten, dass in allen Risikogruppen jeweils sowohl Depressivität den Pfad von elterlicher Psychopathologie auf aggressiv-dissoziales Verhalten mediierte als auch aggressiv-dissoziales Verhalten den Pfad von elterlicher Psychopathologie auf Depression. Die Hypothesen 4 und 5 können damit als bestätigt gelten. Ein differenzierteres Bild ergibt sich erst mit Blick auf die unterschiedliche Varianzaufklärung der Modelle. Für die proaktiv- und die reaktiv- 
aggressive Gruppe war die Varianzaufklärung um ca. 50 \% höher, wenn Depression der Mediator war. Um diesen Befund aufzuklären, wurden zusätzliche, alters- und geschlechtsadjustierte einfache Regressionsanalysen durchgeführt, in denen Depressivität und aggressiv-dissoziales Verhalten aufeinander sowie jeweils auf elterliche Psychopathologie rückbezogen wurden. Deren Ergebnisse legen nahe, dass für beide genannten Risikogruppen elterliche Psychopathologie am stärksten zu einer hohen Varianzaufklärung von aggressiv-dissozialem Verhalten beiträgt: die Varianzaufklärung war jeweils mehr als doppelt so hoch, wenn aggressiv-dissoziales Verhalten allein auf elterliche Psychopathologie rückbezogen wurde als wenn Depression allein auf elterliche Psychopathologie rückbezogen wurde. Depression und aggressiv-dissoziales Verhalten sagten sich bei diesen Analysen jeweils wechselseitig in etwa der gleicher Höhe vorher.

Für mögliche Ursachen von Komorbidität aggressivdissozialen Verhaltens und depressiver Symptome im Sinne der Rolle eines Risikofaktors einer Störung für die jeweils andere ergab diese Studie keine Belege. Vielmehr vermittelten jeweils Depressivität und aggressiv-dissoziales Verhalten wechselseitig die Beziehung von elterlicher Psychopathologie und der jeweils anderen Störung in allen Risikogruppen. Auch die Tatsache, dass aggressivdissoziales Verhalten den Pfad zwischen elterlicher Psychopathologie und Depression vollständig mediierte, kann angesichts der geringen Varianzaufklärung des einfachen Regressionsmodells für elterliche Psychopathologie und Depression und der hohen Varianzaufklärung für jenes mit aggressiv-dissozialem Verhalten allenfalls als schwaches Indiz für eine mögliche Kausalität gelten. Zu dem Postulat einer wahren Komorbidität im Sinne eines bedeutungsvollen, möglicherweise eigenständigen Syndroms (Caron \& Rutter, 1991) stehen die Ergebnisse dieser Analysen jedoch zumindest nicht im Widerspruch. Elterliche Psychopathologie als globales Maß, wie es in der BELLA-Studie erhoben wurde, war ein stärkerer Prädiktor für aggressiv-dissoziales Verhalten als für Depressivität. Dennoch mediierte Depressivität den Pfad auf aggressiv-dissoziales Verhalten und aggressiv-dissoziales Verhalten den Pfad auf Depressivität in allen drei Risikogruppen. Auch standen Depressivität und Aggression in den einfachen Regressionsanalysen wechselseitig in Beziehung, am stärksten in der reaktiv-aggressiven, gefolgt von der proaktiv-aggressiven Gruppe.Amwenigsten intensiv war diese Beziehung in der reaktiv-proaktiv aggressiven Gruppe.

Insgesamt ist die aktuelle Studienlage zur Komorbidität von Aggression und Depression als eigenständiger Störungsentität widersprüchlich. Kopp und Beauchaine (2007) stellten fest, dass komorbid aggressiv/depressive Kinder eine deutlich erhöhte Wahrscheinlichkeit aufwiesen, einen aggressiv-dissozialen Vater und eine depressive Mutter zu haben (sowohl bei einem kategorialen als auch bei einem dimensionalen Klassifikationsansatz) und interpretierten dies vor dem Hintergrund der „Assortative- Cross-Mating“-Hypothese, derzufolge aggressiv-dissoziale Väter und depressive Mütter eine erhöhte Beziehungswahrscheinlichkeit haben (vgl. Kim-Cohen et al., 2005) als Hinweis auf wahre Komorbidität. Ezpeleta et al. (2006) hingegen untersuchten in einer spanischen Inanspruchnahmestichprobe depressive, verhaltensauffällige und komorbide Kinder und Jugendliche im Alter von acht bis 17 Jahren. Sie fanden, dass Komorbidität im klinischen Bild zu viele Ähnlichkeiten mit entweder depressiver oder einer Verhaltensstörung aufweist und kommen daher zu dem Schluss, dass das Postulat einer eigenständigen Störung nicht gerechtfertigt sei. Sie empfehlen, bei komorbider Symptomatik beide Diagnosen zu vergeben.

\section{Schlussfolgerungen für die Forschung und klinische Praxis}

Es überwiegt die Meinung, dass komorbide Aggression/ Depression nicht als eigenständiges Störungsbild aufzufassen ist (z. B. Angold et al., 1999; Jensen, 2003). Dennoch ist die Studienlage nicht eindeutig. Auch in dieser Arbeit ergeben sich diesbezüglich keine klar zu interpretierenden Hinweise. Doch auch wenn die Resultate zur Aufklärung möglicher Mechanismen der Komorbidität nicht eindeutig interpretierbar waren, ergeben sich jedoch einige Aspekte, die Relevanz für die Forschung und die klinische Praxis besitzen. Der Beitrag dieser Studie besteht darin, den Zusammenhang von elterlicher Psychopathologie, aggressiv-dissozialem Verhalten und Depressivität auf funktionale Subtypen von Aggression bezogen zu haben. Augenfällig ist dabei, dass sich vor allem die reaktiv-aggressive Gruppe von beiden anderen Risikogruppen deutlich unterschied. Deskriptiv fiel zunächst der extrem erhöhte Wert für aggressiv-dissoziales Verhalten auf. Auffällig war auch das divergente Muster der bivariaten Korrelationen. Elterliche Psychopathologie war bei der proaktiv- und reaktiv-aggressiven Gruppe höher mit aggressiv-dissozialem Verhalten korreliert als mit Depressivität, in der reaktiv-proaktiven Gruppe korrelierte elterliche Psychopathologie höher mit Depression. 
Die signifikant höheren Werte auf der Depressionsskala für proaktiv-aggressive Kinder sollten Anlass zu weiterer Beobachtung geben. Auch wenn viele klinische Studien proaktive Aggression eher mit späterer aggressivdissozialer Verhaltensauffälligkeit in Verbindung bringen (Fite et al., 2010), gibt es aus der Bullyingforschung durchaus Hinweise auf erhöhte Depressionsneigung bei Bullies (z. B. von Marées \& Petermann, 2009).

Insgesamt scheinen reaktiv-proaktiv aggressive Kinder eine Gruppe mit besonderen Merkmalen zu sein, der in der Forschung und klinischen Praxis bislang zu wenig Aufmerksamkeit zuteil wurde. Zwar wurden auch für die reaktiv-proaktiv aggressive Gruppe die jeweiligen Mediatormodelle signifikant, sie waren jedoch diejenigenmit der geringsten Varianzaufklärung. Für diese Gruppe scheinen weitere Faktoren bedeutsamzu sein, die in dieser Untersuchung nicht aufgeklärt wurden.

Diese Studie weist einige Einschränkungen auf. Zum einen erlaubt der querschnittliche Ansatz keine Aussagen über zeitliche Verläufe. Einflüsse auf die Beziehung zwischen dem mutmaßlichen Risikofaktor elterliche Psychopathologie und der jeweiligen Outcome-Variable wurden über Mediatormodelle getestet, innerhalb derer lediglich unterstellt wird, dass die Vermittlung der Beziehung kausal ist. DieVerwendung eines globalenMaßes für elterliche Psychopathologie erlaubte es nicht, nach unterschiedlichen elterlichen Störungen zu differenzieren. Da der Ausschluss fehlender Werte listenweise erfolgte, ist es denkbar, dass Merkmale von Personenmit fehlenden Werten in systematischer Weise sich von den Personen unterscheiden, die vollständige Angaben aufweisen. Die externe Validität der Studie ist daher eingeschränkt.

\section{Literatur}

Achenbach, T.M. (1991) Manual for the child behaviour checklist/ 4-18 and 1991 profile. Burlington: University of Vermont, Department of Psychiatry.

Angold, A. \& Costello, E. J. (1993). Depressive comorbidity in children and adolescents: Empirical, theoretical, and methodological issues. American Journal of Psychiatry, 7, 669-682.

Angold, A., Costello, E. J. \& Erkanli, A. (1999). Comorbidity. Journal of Child and Adolescent Psychiatry, 40, 57-87.

Baron, R. M. \& Kenny, D. A. (1986). The moderator-mediator distinction in social psychological research: Conceptual, strategic, and statistical considerations. Journal of Personality and Social Psychology, 51, 1173-1182.

Bandura, A. (1973). Aggression: A social learning analysis. Englewood Cliffs: Prentice-Hall. Bandura, A. (1986). Social foundations of thought and action. Englewood Cliffs: Prentice-Hall. Barkmann, C., Erhart, M., Schulte-Markwort, M. \& the BELLA study group (2008). The German version of the Centre for Epidemiological Studies Depression Scale for Children: Psychometric evaluation in a populationbased survey of 7 to 17 years old children and adolescents - results of the BELLA study. European Child and Adolescent Psychiatry 17 (Suppl. 1), 116-124.

Berkowitz, L. (1993). Aggression: Its causes, consequences, and controls. Philadelphia: Temple University Press.

Berg-Nielsen, T. S., Vikan, A. \& Dahl, A. A. (2002). Parenting related to child and parental psychopathology: A descriptive review of the literature. Clinical Child and Adolescent Psychology and Psychiatry, 7, 529-552.

Beyers, J. M \& Loeber, R. (2003). Untangeling developmental relations between depressed mood and delinquency in male adolescents. Journal of Abnormal Child Psychology, 31, 247-266.

Capaldi, D.M. (1992). The co-occurrence of conduct problems and depressive symptoms in early adolescent boys: II A 2-yearfollowup at grade 8. Development and Psychopathology, 4, 125-144. Card N.A. \& Little, T. D. (2006). Proactive and reactive aggression in childhood and adolescence: A meta-analysis of differential relations with psychosocial adjustment. International Journal of Behavioral Development, 30, 466-480.

Caron, C. \& Rutter, M. (1991). Comorbidity in child psychopathology: Concepts, issues and research strategies. Journal of Child Psychology and Psychiatry, 32, 1063-1080.

Cassidy, B., Zoccolillo, M. \& Hughes, S. (1996). Psychopathology in adolescent mothers and its effect on mother-infant interactions: A pilot study. Canadian Journal of Psychiatry, 41, 379-384.

Cohen, P., Brook, J. S., Cohen, J., Velez, N. \& Garcia, M. (1990). Common and uncommon pathways to adolescent psychopathology and problem behavior. In L.N.Robins (Ed.), Straight and devious paths from childhood to adulthood (pp. 242-258). New York: Cambridge University Press. 
Cole, D. A., Martin, J. M. \& Powers, B. (1997). A competency based model of child depression: A longitudinal study of peer, parent, teacher, and self-evaluation. Journal of Child Psychology and Psychiatry and Allied Disciplines, 38, 505-514.

Conger, R., Conger, K., Elder, G., Loenz, F., Simons, R. L. \& Whitbeck, L. (1992). A family process model of economic hardship and adjustment of early adolescent boys. Child Development, 63, 526541.

Crick, N. R. \& Dodge, K. A. (1996). Social information-processing mechanisms in reactive and proactive aggression. Child Development, 67, 993-1002.

Egan, S. K., Monson, T. C. \& Perry, D. G. (1998). Social-cognitive influences on change in aggression over time. Developmental Psychology, 34, 299-309.

Ezpeleta, L., Domènech, J.M. \& Angold, A. (2006). A comparison of pure and comorbid CD/ODD and depression. Journal of Child Psychology and Psychiatry, 47, 704-712.

Fombonne, E.,Wostear,G., Cooper,V., Harrington, R.\& Rutter,M. (2001). The Maudsley long term follow-up of child and adolescent depression. 1. Psychiatric outcomes in adulthood. British Journal of Psychiatry, 179, 210-217.

Franke, G. H. (2002). SCL-90-R. Die Symptom-Checkliste von Derogatis - Deutsche Version (2. Aufl.). Göttingen: Beltz-Test.

Fite, P.J., Raine,A., Stouthamer-Loeber, M., Loeber, R. \& Pardini, D.A. (2010). Reactive and proactive aggression in adolescent males: Examining differential outcomes 10 years later in early adulhood. Criminal Justice and Behavior, 37, 141-157.

Fite, P. J., Stoppelbein, L. \& Greening, L. (2009). Proactive and reactive aggression in a child psychiatric inpatient population. Journal of Clinical Child and Adolescent Psychology, 38, 199-205.

Frick, P. J., Lahey, B. B., Loeber, R., Stouthamer-Loeber, M., Christ, M. A. G. \& Hanson, K. (1992). Familial risk factors to conduct disorder and oppositional defiant disorder: Parental psychopathology andmaternal parenting. Journal of Consulting and Clinical Psychology, 60, 49-55.

Goodman, S. H., Adamson, L. B., Riniti, J. \& Cole, S. (1994). Mothers's expressed attitudes:

Associations with maternal depression and children's self-esteem and psychopathology. Journal of the American Association of Child and Adolescent Psychiatry, 33, 1265-1274.

Groen, G. \& Petermann, F. (2008). Was wirkt in der Therapie von Depression bei Kindern und Jugendlichen wirklich? Kindheit und Entwicklung, 17, 243-251.

Hayes, A. (2009). Beyond Baron and Kenny: Statistical mediation analysis in the New Millenium. Communication Monographs, 76, 408-420.

Helmsen, J. \& Petermann, F. (2010). Soziale Informationsverarbeitung bei körperlich und relational aggressiven Vorschulkindern. Zeitschrift für Kinder- und Jugendpsychiatrie und Psychotherapie, 38, 211-218.

Hirshfeld-Becker, D. R., Carter, P., Micco, J. A., Henin, A., Park, J., Beilin,A., Rosenbaum, J. F.\& Biederman, J. (2008).Disruptive behavior in offspring of parents with major depression: Associations with parental behavoir disorders. Journal of Affective Disorders, 111, 176-184.

Ingoldsby, E.M.,Kohl, O. G.,McMahon, R. J.\& Lengua, L. (2006). Conduct problems, depressive symptomatology and their co occuring presentation in childhood as predictors of adjustment in early adolescence. Journal of Abnormal Child Psychology, 34, 603-621.

Jensen, P. S. (2003). Comorbidity and child psychopathology: Recommendations for the next decade. Journal of Abnormal Child Psychology, 31, 293-300.

Jeske, J., Bullinger, M., Plaß, A., Petermann, F. \& Wiegand-Grefe, S. (2009). Risikofaktor Krankheitsverarbeitung. Zusammenhänge zwischen der Krankheitsverarbeitung einer elterlichen psychischen Erkrankung und der gesundheitsbezogenen Lebensqualität der Kinder. Zeitschrift für Psychiatrie, Psychologie und Psychotherapie, 57, 207-213.

Kiesner, J. (2002). Depressive symptoms in early adolescence: Their relations with classroom problem behavior and peer status. Journal of Research on Adolescence, 12, 463-478.

Kim-Cohen, J., Moffit, T. E., Taylor, A., Pawlby, S. J. \& Caspi, A. (2005). Maternal depression and children's antisocial behavior. Archives of General Psychiatry, 62, 173-181.

Koglin, U. \& Petermann, F. (2008). Inkonsistentes Erziehungsverhalten. Ein Risikofaktor für aggressives Verhalten? Zeitschrift für Psychiatrie, Psychologie und Psychotherapie, 56, 285-291. Kopp,L.M.\& Beauchaine, T. P. (2007). Patterns of psychopthology in the families of children with conduct problems, depression, and both psychiatric conditions. Journal of Abnormal Child Psychology, 35, 301-312.

Kötter, C., Stemmler, M., Bühler,A.\& Lösel, F. (2010).Mütterliche Depressivität, Erziehung und kindliche Erlebens- und Verhaltensprobleme. Kindheit und Entwicklung, 19, 109-118.

Kruse, L. \& Petermann, F. (2010). Erfassung reaktiver und proaktiver Aggression in der Schule: Pilotstudie zur Entwicklung und Validierung eines Fragebogens. Psychologie in Erziehung und Unterricht, 57, 107-118. 
Kurth, B.-M., Kamtsiuris, P., Hoelling, H., Schlaud, M., Dölle, R., Ellert, U., Kahl, H., Knopf, H. et al. (2008). The challenge of comprehensively mapping childrens's health in a nation-wide health survey. Design of the German KiGGS-Study. BMC Public Health, 8, 196.

Marmorstein, N. R. \& lacono, W. G. (2003). Major depression and conduct disorder in a twin sample: Gender, functioning, and risk for future psychopathology. Journal of the American Association of Child and Adolescent Psychiatry, 45, 225-233.

Marmorstein, N. R. \& lacono, W. G. (2004). Major depression and conduct disorder in youth:

Associations with parental psychopathology and parent-child conflict. Journal of Child Psychology and Psychiatry, 45, 377-386.

McConaughy, S. H. \& Achenbach, T. M. (1994). Comorbidity of empirically based syndromes in matched general population and clinical samples. Journal of Child Psychology and Psychiatry, 35, $1141-1157$.

Morrow, T. M.,Hubbard, J.A., McAuliffe, M. D., Rubin,R.M.\& Dearing, K. F. (2006). Childhood aggression, depressive symptoms, and peer rejection: The meditational model revisited. International Journal of Behavioral Development, 30, 240-248.

Murray, L., Kempton, C., Wooglar, M. \& Hooper, R. (1993). Depressed mother's speech to their infants and its relation to infant gender and cognitive development. Journal of Child Psychology and Psychiatry and Allied Disciplines, 34, 1083-1101.

O'Connor, T.G., McGuire, S., Reiss, D., Hetherington, E.M. \& Plomin, R. (1988). Co-occurrence of depressive symptoms and antisocial behaviour in adolescence: A common genetic liability. Journal of Abnormal Psychology, 107, 27-37.

Panak, W. F. \& Garber, J. (1992). Role of aggression, rejection and attributions in the prediction of depression in children. Development and Psychopathology, 4, 145-165.

Petermann, F. (2002). Klinische Kinderpsychologie: Das Konzept der sozialen Kompetenz. Zeitschrift für Psychologie, 210, 175-185.

Petermann, F. \& Petermann, U. (2010). Aggression. Kindheit und Entwicklung, 19, 205-208.

Petermann, F., Petermann, U., Besier, T., Goldbeck, L., Büttner, P., Krause-Leipoldt, C. \& Nitkowski, D. (2008). Zur Effektivität des Trainings mit aggressiven Kindern in Psychiatrie und Jugendhilfe.

Kindheit und Entwicklung, 17, 182-189.

Petermann, U., Reinartz, H. \& Petermann, F. (2002). Ein Explorationsbogen zur Identifizierung individueller Lernwege in der Sozialentwicklung. Zeitschrift für Klinische Psychologie, Psychiatrie und Psychotherapie, 50, 427-457.

Polman, H.,Orobio de Castro, B.,Koops, W., van Boxtel, H. W. \& Merk,W.W. (2007).Ameta-analysis of the distinction between reactive and proactive aggression in children and adolescents. Journal of Abnormal Child Psychology, 35, 522-535.

Preacher, K. \& Hayes A. (2004). SPSS and SAS Procedures for estimating indirect effects in simple mediation models. Behavior Research Methods, Instruments and Computers, 36, 717-731.

Preacher, K. \& Hayes A. (2008). Asymptotic and resampling strategies for assessing and comparing indirect effects in multiple mediator models. Behavior Research Methods, 40, 879-891.

Prinz, U., Nutzinger, D. O. , Schulz, H., Petermann, F., Braukhaus C. \& Andreas, S. (2008). Die Symptom-Checkliste-90-R und ihre Kurzversionen: Psychometrische Analysen bei Patienten mit psychischen Erkrankungen. Physikalische Medizin, Rehabilitationsmedizin, Kurortmedizin, 18, 337343.

Ravens-Sieberer, U., Wille, N., Bettge, S. \& Erhart, M. (2007). Psychische Gesundheit von Kindern und Jugendlichen in Deutschland. Ergebnisse aus der BELLA-Studie im Kinderund Jugendgesundheitssurvey (KiGGS). Bundesgesundheitsblatt Gesundheitsforschung Gesundheitsschutz, 50, 871-878.

Ravens-SiebererU.\& Kurth, B. and the KiGGS study group and the BELLA study group (2008).The mental heath module (BELLA study) within the German Health Interview and Examination Survey for Children and Adolescents (KiGGS). European Child and Adolescent Psychiatry 17 (Suppl 1), 10-21. Rohde, P., Clarke, G. N., Lewihnson, P. M., Seeley, J. R. \& Kaufman, N. K. (2001). Impact on comorbidity on a cognitive behavioral group treatment for adolescent depression. Journal of the American Academy of Child and Adolescent Psychiatry, 45, 795-802.

Rutter, M. (1990) Commentary: Some focus and process considerations regarding effects of parental depression on children. Developmental Psychology, 26, 60-67.

Scheithauer, H., Mehren, F. \& Petermann, F. (2003). Entwicklungsorientierte Prävention von aggressiv-dissozialem Verhalten und Substanzmissbrauch. Kindheit und Entwicklung, 12, 84-99.

Scheithauer, H. \& Petermann, F. (2010). Entwicklungsmodelle aggressiv- dissozialen Verhaltens und ihr Nutzen für Prävention und Behandlung. Kindheit und Entwicklung, 19, 209-217.

Schlack, R. \& Hölling, H. (2007). Gewalterfahrungen von Kindern und Jugendlichen im subjektiven Selbstbericht. Erste Ergebnisse aus dem Kinder- und Jugendgesundheitssurvey (KiGGS).

Bundesgesundheitsblatt Gesundheitsforschung Gesundheitsschutz, 50, 819-826. 
Sourander, A., Jensen, P., Davies, M., Niemela, S., Elonheimo, H., Ristkari, T., Helenius, H., Sillanmäki, L. et al. (2007). Who is at greatest risk of adverse longterm outcomes? The Finnish From A Boy to a Man Study. Journal of the American Academy of Child and Adolescent Psychiatry, 46, 11481161.

Vitaro F., Brendgen, M. \& Tremblay, R.E. (2002) Reactively and proactively aggressive children: antecedent and subsequent characteristics. Journal of Child Psychology and Psychiatry, 43, 495-505. vonMarées,N.\& Petermann, F. (2009). Bullying an Grundschulen. Formen, Geschlechtsunterschiede und psychosoziale Korrelate. Psychologische Rundschau, 60, 152-162.

Whitbeck,L. B.,Hoyt, D. R.,Simons,R. L.\& Conger, R. D. (1992) Intergenerational continuity of parental rejection and depressed affect. Journal of Personality and Social Psychology, 63, 1036-1045.

Wiegand-Grefe, S., Geers, P., Plaß, A., Petermann, F.\& Riedesser, P. (2009). Kinder psychisch kranker Eltern: Zusammenhänge zwischen subjektiver elterlicher Beeinträchtigung und psychischer Auffälligkeit der Kinder aus Elternsicht. Kindheit und Entwicklung, 18, 111-121.

\section{Tabellen und Abbildungen}

Tabelle 1. Deskriptive Statistiken und Korrelationen der Modellvariablen

\begin{tabular}{|c|c|c|c|c|c|}
\hline & & $M(S D)$ & & 1 & 2 \\
\hline Nichtaggressiv $(n=934)$ & $\begin{array}{l}1 \\
2 \\
3\end{array}$ & $\begin{array}{l}6.81(6.16) \\
8.03(5.60) \\
22.88(7.30)\end{array}$ & $\begin{array}{l}\text { Ext. Störung }{ }^{a} \\
\text { Depression } \\
\text { elt. Psychopathologie }\end{array}$ & $\begin{array}{l}.31^{* *} \\
.39^{* *}\end{array}$ & $.30 * *$ \\
\hline Proaktiv-aggressiv $(n=145)$ & $\begin{array}{l}1 \\
2 \\
3\end{array}$ & $\begin{array}{l}9.60(7.91) \\
10.45(7.37) \\
24.37(8.22)\end{array}$ & $\begin{array}{l}\text { Ext. Störung }{ }^{a} \\
\text { Depression } \\
\text { elt. Psychopathologie }\end{array}$ & $\begin{array}{l}.42^{* *} \\
.44 * *\end{array}$ & $.33^{* *}$ \\
\hline Reaktiv-aggressiv ( $n=173$ ) & $\begin{array}{l}1 \\
2 \\
3\end{array}$ & $\begin{array}{l}10.05(8.52) \\
8.40(6.45) \\
25.21(8.15)\end{array}$ & $\begin{array}{l}\text { Ext. Störung }{ }^{a} \\
\text { Depression } \\
\text { elt. Psychopathologie }\end{array}$ & $\begin{array}{l}.46^{* *} \\
.43^{* *}\end{array}$ & $.31 * *$ \\
\hline Reaktiv-proaktiv aggressiv $(n=104)$ & $\begin{array}{l}1 \\
2 \\
3\end{array}$ & $\begin{array}{l}14.30(11.02) \\
9.49(5.49) \\
25.63(7.90)\end{array}$ & $\begin{array}{l}\text { Ext. Störung }^{\mathrm{a}} \\
\text { Depression } \\
\text { elt. Psychopathologie }\end{array}$ & $\begin{array}{l}.29 * * \\
.34 * *\end{array}$ & $.42 * *$ \\
\hline
\end{tabular}

Anmerkungen: a aggressiv-dissoziales Verhalten; ${ }^{* *} p<0.01$.

Tabelle 2. Modellinformationen für die Mediatoranalysen (adjustiert für Alter und Geschlecht) mit (a) externalisierendem (aggressiv-dissozialem) Verhalten und (b) Depression als abhängiger Variable

\begin{tabular}{llllll}
\hline AV & Risikogruppe & $R^{2}$ & Adjustiertes $R^{2}$ & F-Wert & $p$ \\
\hline (a) & & & & & \\
Ext. Verhalten & Nichtaggressiv & .20 & .20 & $F_{(4.929)}=57.95$ & $<.001$ \\
& Proaktiv-aggressiv & .28 & .26 & $F_{(4.140)}=13.86$ & $<.001$ \\
& Reaktiv-aggressiv & .33 & .32 & $F_{(4.168)}=20.95$ & $<.001$ \\
& Reaktiv-proaktiv aggressiv & .18 & .15 & $F_{(4.99)}=5.44$ & $<.001$ \\
\hline \multirow{2}{*}{ (b) } & & & & \\
Depression & Nichtaggressiv & .14 & .13 & $F_{(4.929)}=36.66$ & $<.001$ \\
& Proaktiv-aggressiv & .20 & .18 & $F_{(4.140)}=8.77$ & $<.001$ \\
& Reaktiv-aggressiv & .23 & .21 & $F_{(4.168)}=12.24$ & $<.001$ \\
& Reaktiv-proaktiv aggressiv & .21 & .18 & $F_{(4.99)}=6.67$ & $<.001$ \\
\hline
\end{tabular}

Anmerkung: $\mathrm{AV}=$ abhängige Variable. 
Tabelle 3. Mediatonsergebnisse mit (a) externalisierendem (aggressiv-dissozialem) Verhalten als abhängiger Variable und Depression als Mediator und (b) Depression als abhängiger Variable und externalisierendem Verhalten als Mediator, mit jeweils elterlicher Psychopathologie als unabhängige Variable (adjustiert für Alter und Geschlecht)

\begin{tabular}{|c|c|c|c|c|c|c|c|}
\hline Risikogruppe & AV & MED & $\begin{array}{l}\text { Effekt von UV auf MED } \\
\text { (a-Pfad) }\end{array}$ & $\begin{array}{c}\text { Effekt von MED auf AV } \\
\text { (b-Pfad) }\end{array}$ & $\begin{array}{l}\text { Direkter Effekt } \\
\text { (c'-Pfad) }\end{array}$ & $\begin{array}{l}\text { Indirekter Effekt" } \\
\text { (a x b) }\end{array}$ & Totale Effekte \\
\hline & (a) & & & & & & \\
\hline Nichtaggressiv & Ext. Verhalten & Depression & $.16^{* * *}$ & $.21^{* * *}$ & $.08 * * *$ & $.03^{\dagger}$ & $.11^{* * *}$ \\
\hline Proaktiv-aggressiv & Ext. Verhalten & Depression & $.14^{* * *}$ & $.33^{* * *}$ & $.16^{* * *}$ & $.05^{\dagger}$ & $.20^{* * * *}$ \\
\hline Reaktiv-aggressiv & Ext. Verhalten & Depression & $.12^{* * * *}$ & $.46^{* * * *}$ & $.17 * * *$ & $.05^{\dagger}$ & $.22 * * *$ \\
\hline \multirow[t]{2}{*}{ Reaktiv-proaktiv aggressiv } & Ext. Verhalten & Depression & $.14^{* * *}$ & .39 n.s. & $.17^{*}$ & $.05^{t}$ & $.23^{* * *}$ \\
\hline & (b) & & & & & & \\
\hline Nichtaggressiv & Depression & Ext. Verhalten & $.11^{* * *}$ & $.24^{* * *}$ & $.13^{* * *}$ & $.26^{\dagger}$ & $.16^{* * *}$ \\
\hline Proaktiv-aggressiv & Depression & Ext. Verhalten & $.02^{* * *}$ & $.32^{* * *}$ & $.07 *$ & $.07^{\dagger}$ & $.14^{* * *}$ \\
\hline Reaktiv-aggressiv & Depression & Ext. Verhalten & $.28 * * *$ & $.31^{* * *}$ & $.05 \mathrm{n} . \mathrm{s}$ & $.07^{\dagger}$ & $.12^{* * *}$ \\
\hline Reaktiv-proaktiv aggressiv & Depression & Ext. Verhalten & $.23^{* * *}$ & $.09 \mathrm{n.s}$. & $.12 * * *$ & 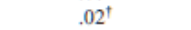 & $.14^{* * *}$ \\
\hline
\end{tabular}

Anmerkungen: $\mathrm{AV}=$ =abhängige Variable; $\mathrm{UV}=$ unabhängige Variable;MED=Mediator; Anzahl der Bootstrap Resamples: jeweils 5000; † signifikanter Punktschätzer $(p<0.05)$; †† Konfidenzintervalle werden im Text berichtet; ${ }^{*} p<0.05 ;{ }^{* *} p<0.01$; ${ }^{* *} p<0.001$; n.s. $=$ nicht signifikant.

Abbildung 1. Mediatormodelle zur Beziehung von elterlicher Psychopathologie und (a) aggressivdissozialem Verhalten mit Depression als Mediator und (b) Depression mit aggressivdissozialem Verhalten als Mediator.

Anmerkung: Die Analysen wurden stratifiziert jeweils für nichtaggressive, proaktiv- aggressive, reaktivaggressive und reaktiv-proaktiv aggressive Kinder und Jugendliche durchgeführt.
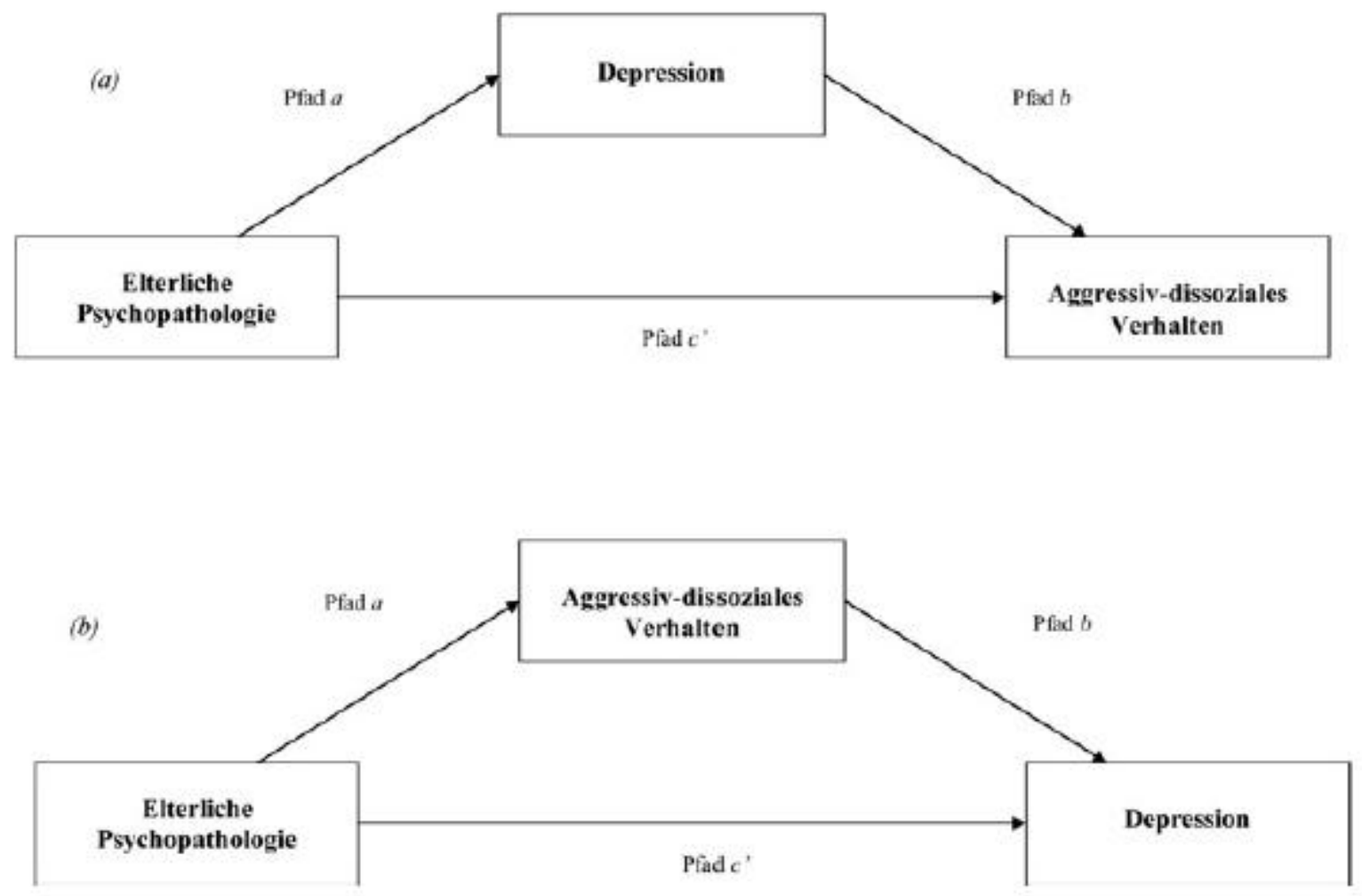